\title{
THE ECOSAR P-BAND SYNTHETIC APERTURE RADAR
}

\author{
Rafael F. Rincon, Temilola Fatoyinbo, Guoqing Sun, K. Jon Ranson, \\ Martin Perrine, Manohar Deshapnde, Quenton Bonds \\ NASA/Goddard Space Flight Center, Greenbelt, MD 20771
}

\begin{abstract}
The EcoSAR instrument is a new concept in Synthetic Aperture Radar for the polarimetric and interferometric measurements of biomass and ecosystem structure. EcoSAR will employ a digital beamforming architecture, a highly capable digital waveform generator and receiver system, and advanced dual-polarization array antennas with an interferometric baseline of $25 \mathrm{~m}$ on the NASA P3 aircraft.
\end{abstract}

\section{Introduction}

EcoSAR is an advanced airborne polarimetric and interferometric P-band SAR instrument in development at NASA/Goddard Space Flight Center through NASA's Instrument Incubator Program (IIP). EcoSAR will provide unprecedented two- and three dimensional fine scale measurements of terrestrial ecosystem structure and biomass. These measurements directly support science requirements for the study of the carbon cycle and its relationship to climate change, recommended by the National Science Foundation's Decadal Survey (2007) and highlited in NASA's Plan for a ClimateCentric Architecture (2010).
EcoSAR will leverage digital beamforming technologies and techniques developed for the L-band Digital beamforming SAR (DBSAR) which have demonstrated advanced SAR imaging for Earth science applications [1][2][3][4]. EcoSAR will employ electronic beamsteering on trasnmit and digital beamforming on receive similar to that proven by DBSAR, permitting the implementation of advanced imaging techniques which overcome problems inherent in conventional SAR systems [1][5][6].

The EcoSAR architecture will allow considerable measurement flexibility such as cross track scanning over a wide range of angles, post processing synthesis of multiple beams, simultaneous measurement over both sides of the flight track, and variable incidence angle. Beams can be reprocessed with different beamwidths and side lobe levels to control swath widths and minimize side lobe contamination. Ambiguities in the receiver signal can be suppressed by post processing by appropriate null-steering of the antenna pattern. 


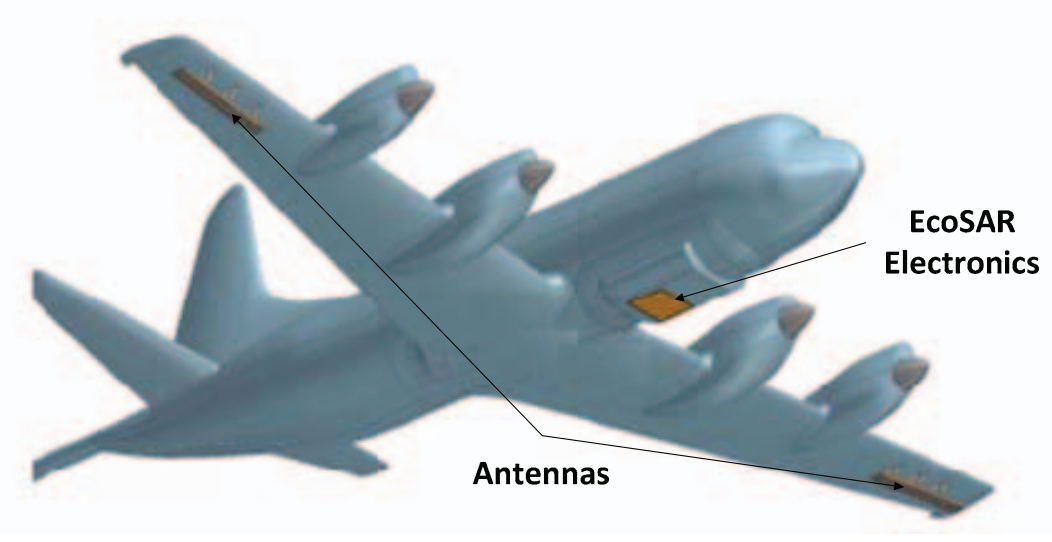

Figure 1 EcoSAR will employ advanced technology and techniques in order to enable polarimetric and interferometric measurements of biomass and ecosystem structure.

\section{$\underline{\text { System Architecture }}$}

EcoSAR will operate at a center frequency of $435 \mathrm{MHz}$ (69 cm wavelength) and feature a fully programmable bandwidth over a 200 $\mathrm{MHz}$ range. Operational modes with slant range resolutions from 5 meters $(30 \mathrm{MHz}$ bandwidth) to 25 meters (6 MHz bandwidth) will be used as a nominal mode in frequency restricted areas, and a science mode with slant range resolutions as fine as $0.75 \mathrm{~m}$ (up to $200 \mathrm{MHz}$ bandwidth) will be employed in authorized or remote areas. EcoSAR's main system characteristics are listed in Table 1.

EcoSAR will perform cross-track interferometry in either "standard" mode or in "ping pong" mode [7] employing two array antennas. The antennas will have dimensions of 3 $\mathrm{m} \times 1 \mathrm{~m}$ and will be mounted under the aircraft wings, as illustrated in Fig1. This configuration will provide a $25 \mathrm{~m}$ baseline in standard mode and a $50 \mathrm{~m}$ baseline in ping pong mode. These baselines are a small fraction of the critical baseline, therefore, minimizing any decorrelation due to the baseline.

The EcoSAR antenna elements will be based on a new microstrip design approach which increases bandwidth and cross-polarization isolation. Antenna element simulations have demonstrated to support $50 \%$ bandwidth and polarization isolation better than $25 \mathrm{~dB}$.

The antenna arrays will be connected to a Radar Electronics Unit (REU) and a Digital 
Table 1 EcoSAR main characteristics

\begin{tabular}{|c|c|c|c|}
\hline Center Frequency & $435 \mathrm{MHz}$ & Pulse Length & 1 usec -50 usec \\
\hline Maximum Bandwidth & $200 \mathrm{MHz}$ & Sub Array Peak Power & 5 Watts \\
\hline Polarization & Full & PRF & $100 \mathrm{~Hz}-10 \mathrm{KHz}$ \\
\hline Polarization Isolation & $>25 \mathrm{~dB}$ & Swath per beam & $4 \mathrm{~km} *$ \\
\hline Single look Azimuth Resolution & $0.5 \mathrm{~m}$ & Number of beams & Multiple \\
\hline Slant Range Resolution & $0.75 \mathrm{~m}$ to $25 \mathrm{~m}$ & Total Number Channels & $36(18 \times 2$ arrays $)$ \\
\hline Noise Equivalent $\sigma^{\circ}$ & $<-41 \mathrm{~dB} *$ & Antenna Dimensions & $3 \mathrm{mx} 1 \mathrm{~m}$ \\
\hline Interferometric baseline & $25 \mathrm{~m}, 50 \mathrm{~m}$ & Antenna Gain & $20 \mathrm{~dB}$ \\
\hline Vertical Accuracy & $1 \mathrm{~m}$ & Number of Subarrays & 9 per antenna \\
\hline
\end{tabular}

* from $8 \mathrm{~km}$ altitude, $35^{\circ}$ incidence angle

Synthesizer and Processing Unit (DSPU)

which will reside in the aircraft fuselage and which will include 18 dual-polarity transmit/receive $(T / R)$ modules, a programmable digital Arbitrary Waveform Generator (AWG) with 36 time-synchronous and phase-locked digital waveform synthesizers (with independent amplitude and phase control), and a reconfigurable data acquisition and real-time processor with 36 independent receive channels, as illustrated in Fig. 2.

The T/R modules will include both transmit and receive calibration loops which when combined with the AWG will allow optimization of the antenna beam, and pulse compression performance by dynamic measurement and removal of nonlinearities and phase and amplitude variations in the transceiver.

The EcoSAR architecure will suport full polarimetric operation and a hybrid polarity operation (i.e., transmit circular, receive horizontal and vertical polarizations) for the retrieval of the full backscattering matrix (Raney, 2007). Orthogonal polarity waveform generation techniques will also be used to implement RFI mitigation techniques.

\section{Conclusion}

By building on the past successes of DBSAR, ECOSAR will provide a highly adaptive dual polarization interferometric SAR instrument that will greatly improve the retrieval of biomass and ecosystem structure. 


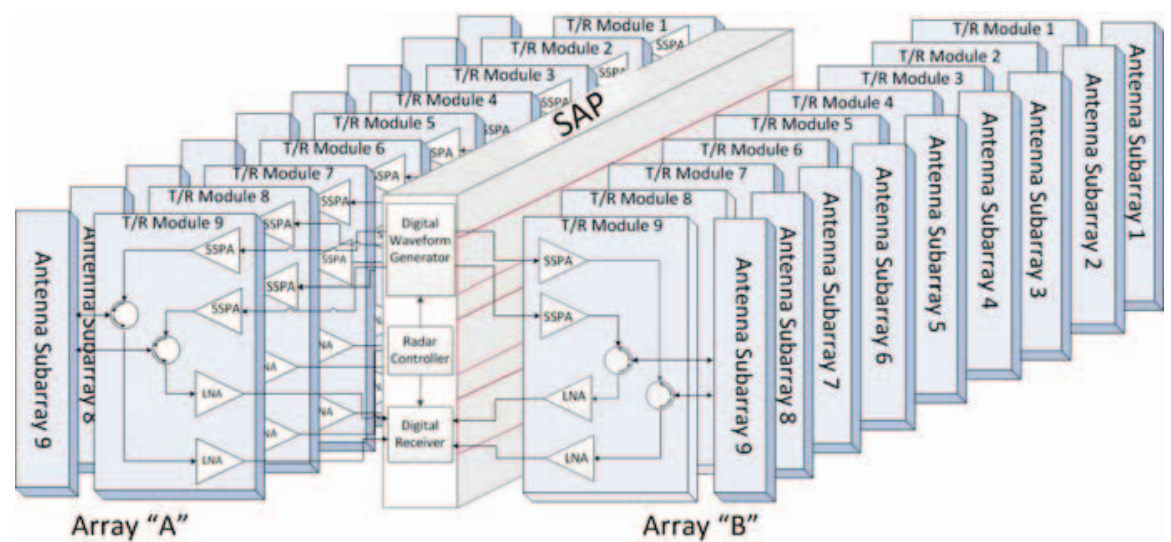

Figure 2 EcoSAR architecture supports polarimetric and interferometric measurements required to characterization of ecosystems and the quantification of biomass.

\section{$\underline{\text { References }}$}

[1] Rincon, R, M. Vega, M. Buenfil, A. Geist, L. Hilliard, and P. Racette, "NASA's Digital Beamforming SAR (DBSAR)", 2011," IEEE Transactions on Geoscience and Remote Sensing, Accepted for publication.

[2] Rincon, R, M. Vega, M. Buenfil, A. Geist, L. Hilliard, and P. Racette, "DBSAR's First Multimode Flight Campaign”, 2010 European Radar Conference, EUSAR 2010.

[3] Rincon, R.F., "Reconfigurable L-band radar", 2008, Proceedings of the 5th European Radar Conference. EuRAD 2008. vol., no., pp.104-107.

[4] Rincon, R., "NASAS Digital Beamforming Synthetic Aperture Radar", 2008, IGARSS 2008 Proceedings, Boston, Massachusetts, July 6-11.
[5] G. Krieger, N. Gebert, and A. Moreira, "Multidimensional Waveform Encoding: A New Digital Beamforming Technique for Synthetic Aperture Radar Remote Sensing", Trans. Geosci. Remote Sensing, vol. 46, No. 1, pp. 31 - 46, Jan 2008.

[6] M. Younis, C. Fischer, and W. Wiesbeck, "Digital Beamforming in SAR System", Trans. Geosci. Remote Sensing, vol. 41, No. 71, pp. 1735 - 1739, Jul. 2003.

[7] P. A. Rosen, S. Hensley, I. R. Joughin, F. K. Li， S. N. Madsen, E. Rodríguez, and R. M. Goldstein,"Synthetic Aperture Radar Interferometry", Proc. IEEE, vol. 88, no. 3, pp. 333- 382, Mar. 2000.

[8] Raney, R. K., "Hybrid-Polarity SAR Architecture, 2007," IEEE Transactions on Geoscience and Remote Sensing, vol. 45, No. 11, pp. 3397- 3404. 